\title{
New information about practical application of the modified magnetoelastic method
}

\author{
Tomáš Klier ${ }^{1}$, Tomáš Míčka ${ }^{1}$, Michal Polák ${ }^{2, *}$, Tomáš Plachý ${ }^{2}$, Milan Hedbávný ${ }^{3}$, and \\ Lenka Krejčíková ${ }^{3}$ \\ ${ }^{1}$ Pontex, spol. s r. o. Bezová 1658, 147 14, Prague 4, Czech Republic \\ ${ }^{2}$ Czech Technical University in Prague - Faculty of Civil Engineering, Thákurova 7, 166 29, \\ Prague 6, Czech Republic \\ ${ }^{3}$ Freyssinet CZ a. s., Zápy 267, 250 01, Brandýs nad Labem, Czech Republic
}

\begin{abstract}
In the technical practice there is very often a need of axial force determination in the important structural elements of a building during its construction or operational state with adequate precision. The magnetoelastic method is one of the five experimental techniques usually used for that purpose in civil engineering practice. The modified magnetoelastic method is especially aimed on experimental evaluation of the axial forces in the prestressed steel reinforcements on prestressed concrete structures and it is usable not only for newly built structures but in particular for existing ones. New information and knowledge about practical application of the new approach based on the magnetoelastic principle is introduced in the paper. The results of three experiments are summarized, which were realized on the full locked cable PV 150 standardly used as a cable stay strand, on the MUKUSOL threadbar 15FS 0000 generally applied as a temporary prestressed reinforcement and on some prestressed tendons of an existing concrete road bridge, which is about thirty years old.
\end{abstract}

\section{Introduction}

The paper presents the latest knowledge and experience about the modified magnetoelastic method and brief results of its practical applications carried out by authors in the last year.

The fundamental motivation of the research work of the authors is to research and develop a new approach based on the "magnetoelastic" physical principle and to transfer the obtained knowledge to practical applications. This paper follows up their previously published activities, especially in [1-3].

The magnetoelastic method is based on the physical principle that is functional only on structural elements made of ferromagnetic materials, as are steel prestressed rods, steel wires and steel tendons for example. The new approach is in particular proposed for the experimental determination of axial forces in steel prestressed reinforcements on existing civil engineering structures made of prestressed concrete. This task is very common nowadays in technical practice, especially on bridges, but other methods are generally not usable for that purpose or the difficulties with their applications are more significant and the

*Corresponding author: polak@fsv.cvut.cz 
uncertainties of results are usually greater. The new approach is, however, applicable for the same reason also on newly constructed structures without any substantial modifications and if any modification is finally implemented it deals generally with a simplification of the procedure compared to the existing ones.

In the Czech Republic and as well as in other countries of the world, there are many civil engineering structures and also building ones on which important structural elements are applied that are loaded by large tensile forces, as are suspension cables and vertical suspenders of suspension bridges, stays of cable-stayed bridges, support cables of tensile fabric structures, prestressed reinforcements of prestressed concrete structures, ground and rock anchors, for instance. The load bearing capacity of the whole structure is very often influenced significantly by these elements.

The tensile force in these elements generally varies in time and this fluctuation may be substantial depending on actual variable load, temperature of the element and the whole structure, creep of concrete or losses of prestressing force, for instance. The information about actual value of the tensile force, that is accurate enough, is necessary and important for exact assessment of the reliability of the whole structure in any of its life cycle, as are its individual construction stages, its start to operation, monitoring of its construction conditions in the course of its service, preparation of its reconstruction, et cetera.

In civil engineering practice, five experimental approaches are generally applied to determine the axial tensile forces in the significant structural elements. The first approach is the direct measurement of the axial force by a pre-installed load cell, second one is the technique based on a strain measurement with strain gauges, the third one is the vibration frequency method $[4,5]$, the fourth approach is the force determination in a flexible structural element based on the relation between the transverse force and the caused transverse displacement [6, 7] and the fifth one is the magnetoelastic method [1, 2, 3, 8, 9].

All above mentioned techniques have both their advantages and disadvantages. The specific approach suitable enough for a particular practice application has to be chosen carefully according to specific conditions and requirements, as are applicability of the chosen method for the application and uncertainties of obtained experimental results, for example. According to the authors opinion, only three of the above stated approaches are useful for some new experiment starting on an existing structure that is already in service at that time.

The vibration frequency method is the first one, but it is only useable for structural elements with both their relatively long free vibrating length and clearly enough defined their boundary conditions. It means the method is not applicable reasonably on existing concrete structures where the reinforcements are permanently embedded in concrete.

The method based on the relation between the transverse force and the transverse element displacement is the second one, but it is sensibly applicable only for significantly flexible elements with a relatively long free length. The measurable transverse displacement increases considerably the tensile force in the substantially short element. It means the method is applicable with difficulty for the embedded prestressed reinforcement which is uncovered for the experiment purpose only minimally. Moreover, in the case, where the examined prestressed tendons is assembled from several strands or wires, the experiment is limited to the top ones only.

The third method is a new approach based on the magnetoelastic method. The outcomes of three separate experiments are stated below in the text.

\section{Experiment realized on the full locked cable PV 150}

The basic goal of the experiment described in this paragraph was to verify the applicability of the modified magnetoelastic method for a specific sort of the steel cables, the full locked cables, used very often in construction practice. There was some sort of uncertainty, if the 
new approach based on the magnetoelastic method is sensitive enough for its meaningful practical applications on the full locked cables because of their special structure. The core of these cables is composed from several layers of wires with a circular cross section and the outer layers are formed from Z-shaped wires. The full locked cable PV 150 (see Fig. 1) made by firm Pfeifer Seil- und Hebetechnik GmbH was used for the experiment. The diameter of the cable PV 150 is $40 \mathrm{~mm}$ and its characteristic breaking load is $1520 \mathrm{kN}$.
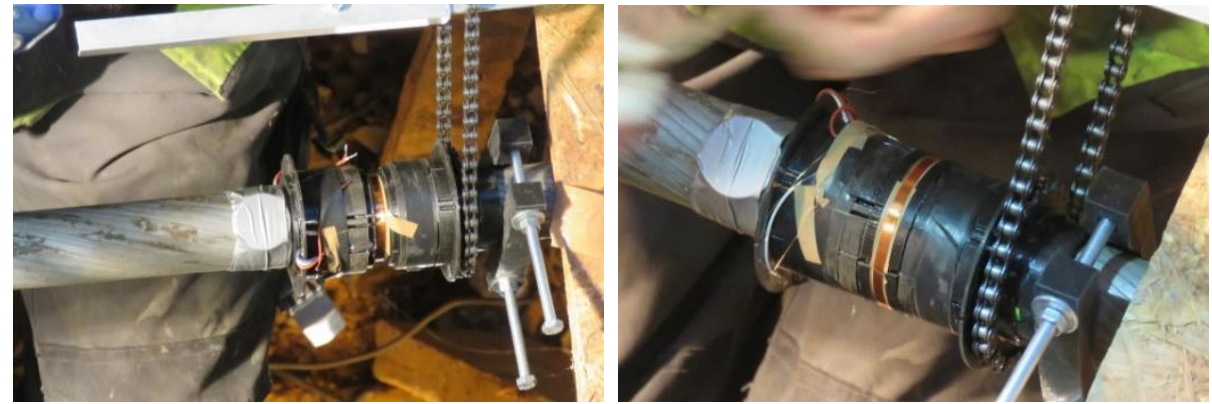

Fig. 1. The cable PV 150, the sensor body mounted on the cable, the made secondary coil 1 (left) and the made secondary coil 2 (right).
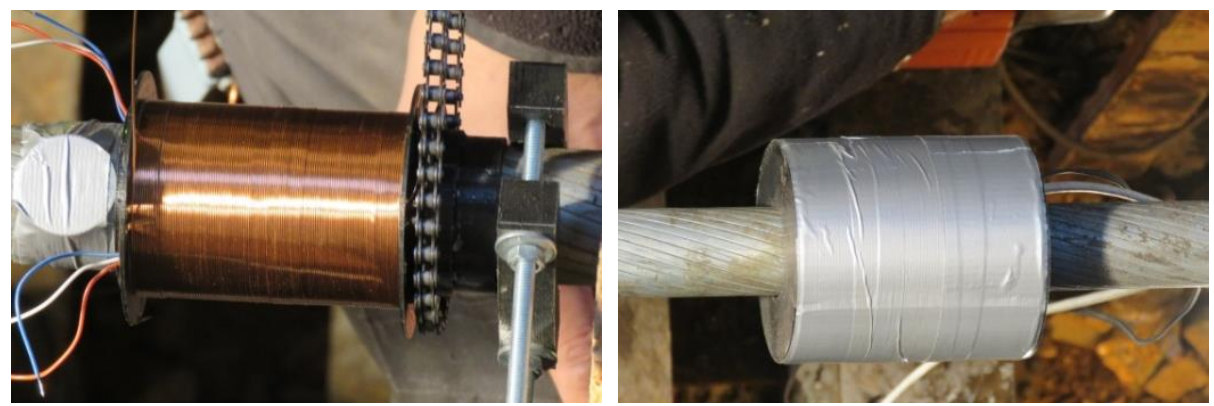

Fig. 2. The primary coil in the course of its production process (left) and the completed sensor with steel shield (right).

The fully equipped elastomagnetic sensor (hereinafter EM sensor) introduced in detail in [1] was applied in the course of the experiment. Its fundamental components were the sensor body made by a 3D printer (see Fig. 1), the primary coil (see Fig. 2) (the controlled magnetic field source), the secondary coil 1 (see Fig. 1) (a sensor of magnetic flux that is closely related to the magnetic induction "B" in the measured cable cross section), the system of two Hall sensors and the secondary coil 2 (see Fig. 1) (two independent sensors of magnetic field intensity "H" in a measured cable section) and steel shield of the EM sensor (see Fig. 2) (a sensor protection against magnetic influences from its surroundings).

The fundamental body of the sensor was glued together on the cable from two individual parts, then the secondary coil 1 was wound up from thin copper wire using the forced sensor body rotation (see Fig. 1), next the partial part of the sensor body was assembled that covered the secondary coil 1, then the secondary coil 2 was wound up (see Fig. 1) and it was covered subsequently by a thin insulation layer. As the next step of sensor production, the primary coil was wound up from thicker copper wire. The steel shield of the EM sensor was installed finally (see Fig. 2).

In the course of the own experiment, the investigated cable was anchored in a stand and loaded by the prestressing force produced by a calibrated tension hydraulic jack. The studied cable was loaded twice in nine force steps, as it can be seen from Fig. 3 and Fig. 4. 
For each used force step, the hysteresis loop was measured and determined, results presented in Fig. 3 and Fig. 4 were evaluated based on the experimental data about magnetic field intensity "H" measured by two applied Hall sensors. However, the experiment demonstrated, the experimental information about " $\mathrm{H}$ " can be obtained from the second coil 2 in the similar quality.

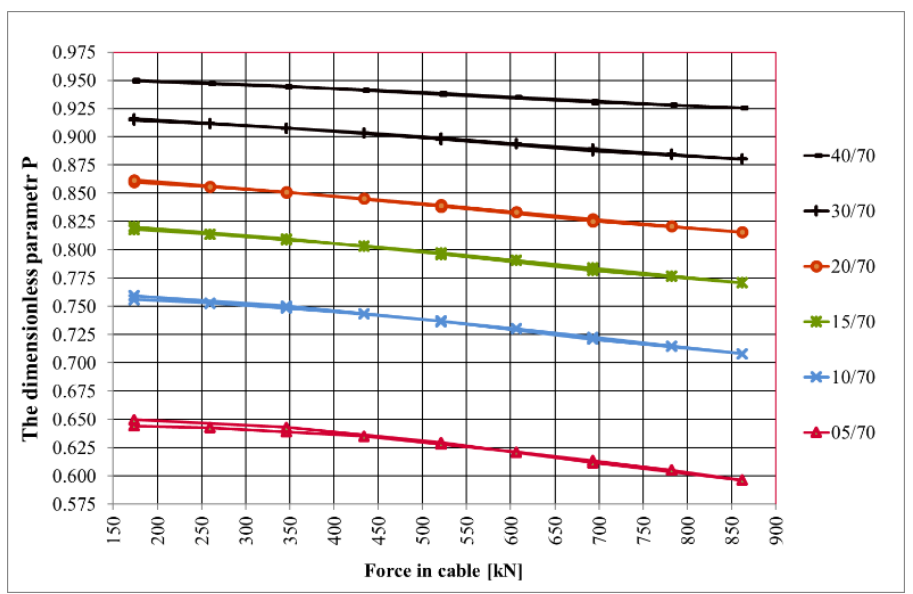

Fig. 3. The cable PV 150 - comparison of the different resultant dimensionless parameter P.

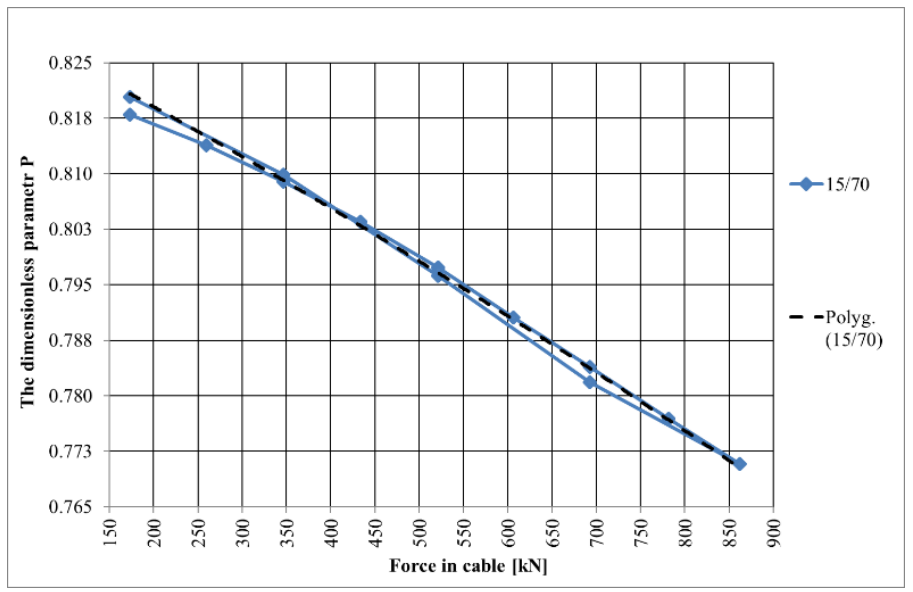

Fig. 4. The cable PV 150 - the relation between the force in the observed cable and the chosen resultant dimensionless parameter $\mathrm{P}$ 15/70.

The dimensionless parameter P shown in Fig. 3 and Fig. 4 is used to convert a measured shape of the hysteresis loop, that depends on the actual force magnitude, to a simple numeric value. The fractions in Fig. 3 describe points of the hysteresis loop taken into account as the key node points for the conversion. The numerator of the fractions is the most important value for evaluation of the parameter $\mathrm{P}$ indicating the level of the magnetic field intensity " $\mathrm{H}$ " in the main node point. The lower values of this indicator indicate the preference of the portion of the hysteresis loop close to the remanence (the intersection with the vertical axis in the $\mathrm{B}-\mathrm{H}$ curve). On the contrary, its higher values prefer the loop portion near to the saturation. The more exact definition of the parameter $\mathrm{P}$ is an industrial secret.

In the course of the described verification experiment, several various dimensionless parameters $\mathrm{P}$ were evaluated that represented in a simple way the character and shape of measured hysteresis loops. The sensitivity of the evaluated parameters $\mathrm{P}$ to the prestressing 
force was studied (see Fig. 3). For further analysis, the parameter P "15/70" was chosen as the most suitable one because of its sufficient sensitivity and satisfactory stability of obtained results (see Fig. 3).

For the chosen parameter $\mathrm{P}$ " $15 / 70$ " the detailed analysis of its sensitivity to the prestressing force in the cable was carried out, the results of this analysis are shown in Fig. 4. The resultant regression fitting curve (the doted curve in Fig. 4) was determined using methods of mathematical analysis and statistics. The maximal differences between the measured values and the resultant curve are about $2.5 \%$ of the defined characteristic breaking load.

Based on the evaluated data, it can be stated, it is possible to determine the prestressing forces in the full locked cables using the new approach based on the magnetoelastic method. The sensitivity of the EM sensor in this case is sufficient and behavior of the EM sensor is similar to ones created for other investigated prestressed elements.

\section{Experiment realized on the MUKUSOL threadbar 15FS 0000}

The experiment described in this chapter was realized on the MUKUSOL threadbar 15FS 0000 made by Dywidag company. Its bar diameter is $15 \mathrm{~mm}$, the thread diameter is 17 $\mathrm{mm}$ and its breaking load is $170 \mathrm{kN}$.

The authors had formerly carried out the similar experiment on the same type of the threadbar and they published the obtained results subsequently in reference [3]. The applicated EM sensor was then made without the secondary coil 2 and the system of Hall sensors was used as the transducer of magnetic field intensity "H". At that time, it was stated that the screw thread of the bar influences significantly negatively the experiment and that the screw thread has to be removed from the bar surface in the proximity of the cross section, where the magnetoelastic sensor would be installed, for obtaining the acceptably accurate results by the magnetoelastic method.

The basic goal of the newer experiment was to verify the usability of the EM sensor equipped with the secondary coil 2 for tests on the prestressed steel bars despite the results mentioned in [3].

The both secondary coils used in the EM sensor were made with the same length as the screw thread pitch of the investigated threadbar for the purpose to reduce the negative influence of the threads.

The EM sensor body was divided into seven parts printed on a $3 \mathrm{D}$ printer during its production (see Fig. 5). Firstly, the secondary coil 1 was wound up on the fundamental part of the sensor body using the forced sensor body rotation. The winding of the coil was made from thin copper wire. The two partial parts of the sensor body, that covered the secondary coil 1 , were then assembled on the sensor body, the secondary coil 2 was wound subsequently on them and it was covered afterward by next two partial parts of the sensor body. As the next step of sensor production, the primary coil was wound up from thicker copper wire and the last two parts of the sensor body were glued together with the fundamental part (see Fig. 5). The steel shield of the EM sensor was installed finally (see Fig. 6). The general view on the experiment arrangement with the produced EM sensor installed on the analysed prestressed bar is shown in Fig. 6.

As it was explained above, the construction of the EM sensors, that were applied during the tests described in this paper, was changed compared to the previous experiments introduced in [1-3] and the type of the control and data acquisition system was changed too.

The previously used type of the control and data acquisition system was equipped by a power amplifier, that allowed precise control of the excitation current flowing into the primary coil within its performance capabilities. This feature enabled significant slowdown of excitation impulses with the objective to reduce a negative influence of eddy currents 
flowing in the ferromagnetic material during quick changes of an electromagnetic field. However, the slowdown of the excitation impulses led to higher energy demands on their realization. As a consequence of this fact the EM sensors were warming up considerably and it affected subsequently temperature changes of the observed prestressed elements in the course of the experiments and this matter of fact influenced negatively the obtained results. It is necessary to consider these negative influences during preparation of a particular experiment and reduce them, to the extent possible, to an acceptable level in the phase of the EM sensor design and the selection of the excitation current source.

For the experiment described in this chapter, the new method was used for generation of the excitation current. The energy accumulated in some pre-charged capacitor batteries was applied for this purpose. However, the possibilities of regulation of the current time behavior are much more limited compared to the approach described in the previous paragraph. The influence of eddy currents occurred in the observed ferromagnetic material was more pronounced due to the speed up excitation current impulse.
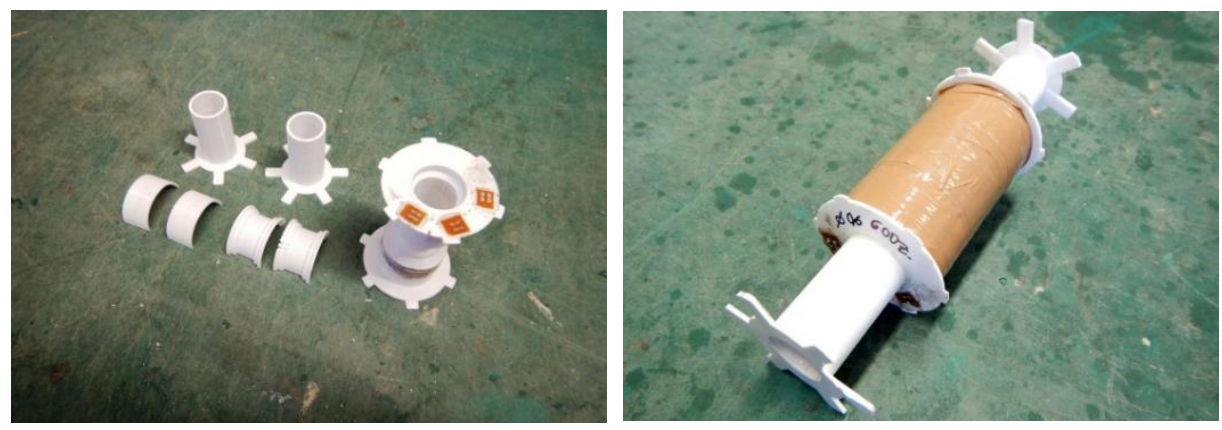

Fig. 5. The MUKUSOL threadbar 15FS 0000 - the disassembled sensor body (on left) and the finished sensor without steel shield (on right).
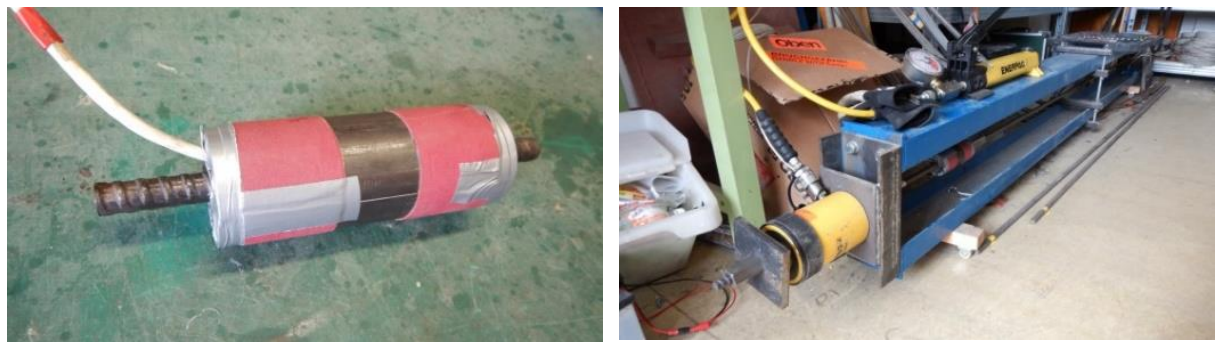

Fig. 6. The MUKUSOL threadbar 15FS 0000 - the completed sensor with steel shield (on left) and the general view on the experiment arrangement (on right).

Eddy currents were obviously generated also during the experiment realized on the full locked cable PV 150 that is described in the previous chapter. However, their influence increased quite fundamentally in the curse of the experiment on the massive MUKUSOL threadbar 15FS 0000. From a magnetic point of view, the cable PV 150 was composed from smaller parts that were separated from each other and this feature reduced significantly the development of eddy currents.

The disturbing influence of eddy currents on the usual procedures that were used to evaluate the results of earlier experiments is clearly evident in Fig. 7. Significantly different dependencies between the resultant dimensionless parameters $\mathrm{P}$ and the prestressing force were found during the experiment on the threadbar compared to the one realized on the cable PV150 (see Fig. 3). The zero or even negative sensitivity was found for some dimensionless 
parameters P. This abnormality appears to be related to the above mentioned negative influence of eddy currents.

Six dimensionless parameters $\mathrm{P}$ were carefully analyzed for the MUKUSOL threadbar 15FS 000 (see Fig. 7). The dimensionless parameter P " $20 / 100$ " was finally chosen as the most suitable one for further analysis of the threadbars and another prestressed bars because of its maximal sensitivity to the prestressing force and its minimal disturbance by negative influences (see Fig. 7).

For the chosen parameter P "20/100" the detailed analysis of its sensitivity to the prestressing force in the bar was carried out, the results of this analysis are shown in Fig. 8. The maximal differences between the measured values and the resultant regression fitting curve (the polynomial of degree 2 ) are about $2 \%$ of the defined characteristic breaking load.

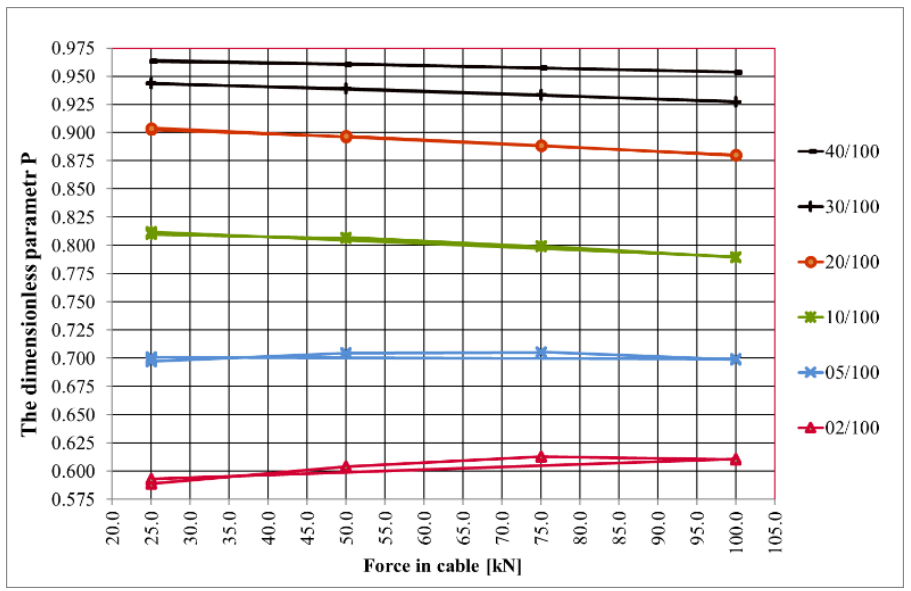

Fig. 7. The MUKUSOL threadbar 15FS 0000 - comparison of the different resultant dimensionless parameter $\mathrm{P}$.

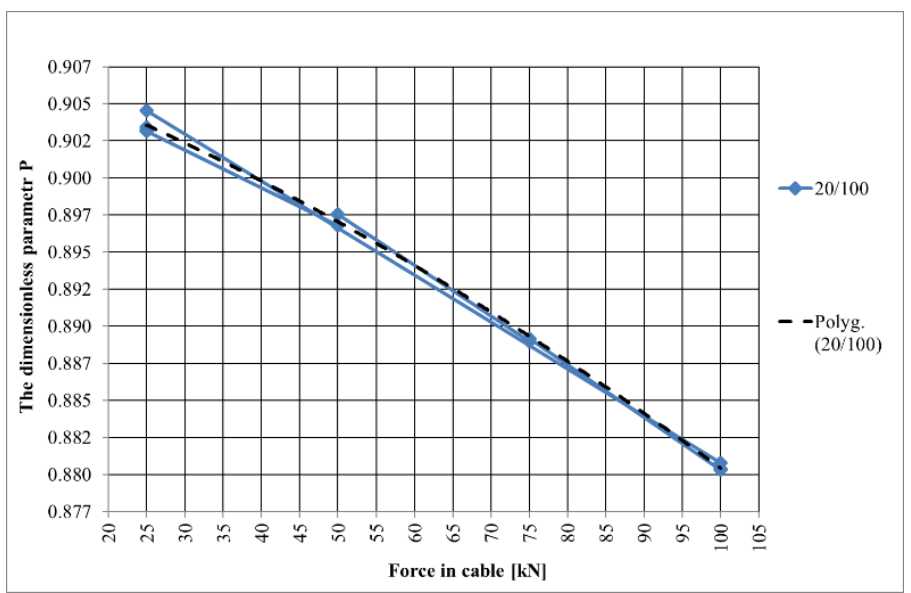

Fig. 8. The MUKUSOL threadbar 15FS 0000 - the relation between the force in the observed bar and the chosen resultant dimensionless parameter P 20/100.

The influences of different positions of the EM sensor on the observed threadbar and also sensor rotations were observed and analyzed for one selected constant force value. These effects caused the maximal mutual differences between evaluated results and also the resultant regression fitting curve at about one percent of the threadbar breaking load. 
Based on the evaluated data, it can be stated, it is possible to determine the prestressing forces in the threadbars and another prestressed bars. However, the higher susceptibility to parasitic effects of eddy current has to be considered by preparation and design of an experiment realized on steel prestressed bars. These effects could be substantially compensated using calibration of a particular experiment system including a specific EM sensor, a particular control and data acquisition system and a specific type of the analyzed prestressed bar.

The lower sensitivity of the EM sensor for the prestressed bars is influenced by their lower working range of a normal stress that reaches about $600 \mathrm{MPa}$. This stress is approximately twice as large for other steel prestressed elements as for prestressed strands, cables and wires.

\section{Experiment realized on an existing road bridge}

The experiment described in this chapter was focused on an existing multi-span road bridge constructed from prestressed concrete which is about thirty years old. The fundamental objective was to determine actual tension forces in six selected prestressed tendons.

The longitudinal prestressing of the bridge was applied by means of post-tensioning tendons that were assembled from twelve strands Lp 15.5. The tendons are led inside steel ducts that are permanently embedded in the monolithic concrete construction and they are curved both in the longitudinal and in the transverse direction. During the construction of the bridge, the tendons were joined by couplers in the working joints and they were injected with cement grout after tensioning of the strands.

The six positions where the experiment on the prestressed tendons was carried out were selected so that it was possible to compare the actual values of the prestressing forces in the chosen tendons considering the influence of visually apparent defects on the concrete surface. Some marks of long-term water penetration, poor quality work and irregular effects of concrete creep were taken into account for example.

In the course of the experiment, the EM sensors were installed on the selected observed prestressed tendons (see Fig. 9). A very similar way of sensor production was used as in the case of the full locked cable PV 150 which is described in the Chapter 2. However, as can be seen from comparing Fig. 9 and Fig. 2, the EM sensors were larger than the one used on the cable PV 150. All installed EM sensors were fully equipped [1]. It should be underlined, both the secondary coil 2 and the system of two Hall sensors were applied.

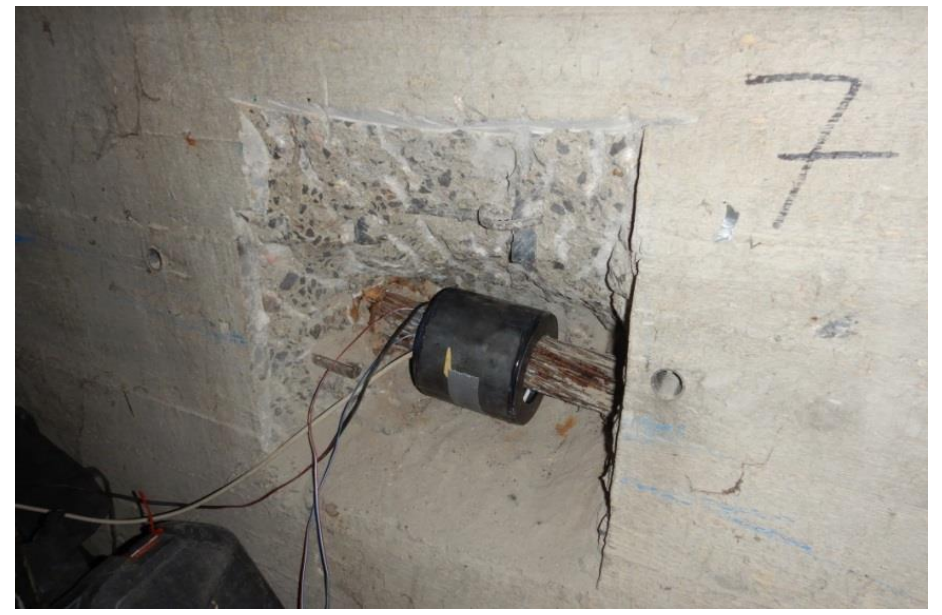

Fig. 9. The EM sensor installed on the tendon that is assembled from twelve strands Lp 15.5. 
Each observed cross section of a prestressed tendon is unique because the mutual relative positions of the strands forming the tendon vary in longitudinal direction, for example due to the tendon curvature. For this reason, it is necessary to use the developed methodology for the application of the modified magnetoelastic method on existing structures which is described in more detail in [1]. The force evaluation by the methodology consists of four main steps. The first one is the as accurate as possible measurement of the actual geometric shape of the tendon in the observed cross-section. The second step is theoretical modeling of the measured geometric shape of the tendon in a 3D software for the electromagnetic field analysis. The third one is the in situ observation of the electromagnetic behavior of the EM sensor installed on the tendon. The fourth one is the application of the available calibration curves that were obtained in laboratory tests realized for strand type (or wire type) from which the analyzed tendon is assembled.

The summary of the determined prestressing forces in the observed selected tendons is shown in Table 1. Two facts are obvious from the published results.

Firstly, it is possible to compare the analyzed prestressing forces that were determined more times in two positions EM3 and EM6. The experiments were carried out repeatedly on the same two tendons, however, their geometric shapes were different. The EM sensors were shifted on an uncovered section of the tendons into some different locations and also position of Hall sensors in the cross section was changed. The comparison of the obtained results in individual positions on the tendon EM3 (the positions EM3a, EM3b and EM3c) and on the tendon EM6 (the positions EM6a and EM6b) shows a partial uncertainty of the evaluated results that includes also influence of software modeling of the analyzed tendons. The difference between compared results is about $1 \%$ of the determined force.

Table 1. The summary of the determined prestressing stresses in the selected tendons.

\begin{tabular}{|c|c|c|}
\hline $\begin{array}{c}\text { Selected } \\
\text { tendon }\end{array}$ & $\begin{array}{c}\text { Dimensionless } \\
\text { parameter P }\end{array}$ & $\begin{array}{c}\text { Determined } \\
\text { tension stress } \\
{[\mathrm{MPa}]}\end{array}$ \\
\hline EM1 & 0.8807 & 666 \\
\hline EM3a & 0.8651 & 878 \\
\hline EM3b & 0.8656 & 871 \\
\hline EM3c & 0.8644 & 888 \\
\hline EM4 & 0.8785 & 696 \\
\hline EM5 & 0.8933 & 495 \\
\hline EM6a & 0.8792 & 687 \\
\hline EM6b & 0.8792 & 687 \\
\hline EM7 & 0.9015 & 384 \\
\hline
\end{tabular}

Secondly, it is possible to compare mutually the individual determined prestressing forces in six investigated positions. The determined forces in the individual analyzed tendons differed much more than it was originally expected in the course of the experiment preparation. The determined prestressing forces in two tendons EM5 and EM7 reached only about $50 \%$ of the maximal evaluated force value. These two tendons were subsequently analyzed in detail and it was found out that these tendons were significantly weakened by corrosion nearby the installed EM sensors and that the corrosion development was irregular. The low quality of the injection of cement grout on these two tendons caused that the low value of the prestressing force was distributed from locations affected by corrosion to the observed cross sections of the tendons.

The described experiment verified the possibility of additional installation and utilization of the EM sensors on the tendons on existing prestressed concrete structures. The installation of the sensor is connected with very laborious activity which goal is a precise local demolition of concrete in the selected sensor position (see Fig. 9). The opening required for EM sensor 
installation allows also good access to the prestressing reinforcement for corrosion engineers to do necessary revision. In the case of lower quality of the injection of cement grout, the EM sensor may reveal significant corrosion of tendons even outside the created opening.

\section{Conclusions}

According to the authors' opinion, the new approach based on the magnetoelastic method is currently the most suitable technique for experimental determination of the tensile stress or force in prestressed reinforcements on existing concrete structures where the reinforcements are permanently placed inside the surrounding concrete. It is, indeed, the truth that the concrete has to be removed in the position chosen for installation of the EM sensor, however, the required opening could be relatively small, and then its influence on the structure may be neglected.

The realized experiment shows that is possible to determine the prestressing forces in the full locked cables using the new approach based on the magnetoelastic method. The sensitivity of the EM sensor in this case is sufficient and similar to ones determined for other investigated prestressed elements.

It is possible to determine the prestressed forces in the threadbars and another prestressed bars using the modified magnetoelastic method and fully equipped EM sensor. However, the higher susceptibility to parasitic effects of eddy current has to be considered by preparation and design of an experiment realized on steel prestressed bars.

The experiment carried out on the existing bridge structure verified the possibility of additional installation and utilization of the EM sensors on the prestressed reinforcements on existing prestressed concrete structures. In the case of lower quality of the injection of cement grout, the EM sensor may reveal significant corrosion of tendons even outside the created opening.

The results presented in this paper are outputs of the research project FV 30457 "Utilization of a Magnetoelastic Method for Increasing the Reliability and Durability of Existing and Newly Built Prestressed Concrete Structures" supported by Ministry of Industry and Trade of the Czech Republic.

\section{References}

1. T. Klier, T. Míčka, M. Polák, T. Plachý, M. Hedbávný, R. Jelínek, F. Bláha, Acta Polytechnica CTU Proceedings, 15, 46-50 (2018)

2. Klier, T., Míčka, T., Plachý, T., Polák, M., Smeták, T., Šimler, M., MATEC Web Conf., 107, 00015 (2017)

3. Klier, T, Míčka, T, Polák, M., Plachý, T., Šimler, M, Smeták, T., Proceedings of the 55th Conference on Experimental Stress Analysis 2017, 122-132 (2017)

4. M. Polák, T. Plachý, Procedia Engineer., 48, 578-582 (2012)

5. M. Polák, T. Plachý, Appl. Mech. Mater., 732, 333-336 (2015)

6. P. Fajman, M. Polák, Proceedings of the 50th Annual Conference on Experimental Stress Analysis, EAN 2012, 61-64 (2012)

7. P. Fajman, M. Polák, J. Máca, T. Plachý, Appl. Mech. Mater., 486, 189-194 (2014)

8. A. M. Sarmento, A. Lage, E. Caetano, J. Figueiras, Proceedings of the 6th International Conference on Bridge Maintenance, Safety and Management IABMAS 2012, 914-921 (2012)

9. M. Chandoga, P. Fabo, A. Jaroševič, Proceedings of the 2nd fib Congress, 674-675 (2006) 